\title{
Increasing burden of diabetes in Bangladesh and the role of information technology
}

\author{
Sheikh Mohammed Shariful Islam* \\ The George Institute for Global Health, Research Tutor, MD Program, Sydney Medical School, University of Sydney, Missenden Road, Camperdown, NSW 2050, \\ Australia
}

In recent years, non-communicable diseases (NCDs) such as diabetes, cardiovascular diseases, respiratory disease and cancers have increased in epidemic proportions globally. Around $80 \%$ of deaths due to NCDs occur in low-and-middle-income countries (LMICs), where the health systems are inadequate to provide the essential services ${ }^{1}$. The epidemiological transition and double burden of diseases in developing countries might be resulting from increasing age of the population, increase in average lifespan, decrease in childhood infections and death, rural-urban migration, and adoption of unhealthy lifestyle such as sedentary work habits, diets rich in fats, carbohydrate, and salt among others [1]. Diabetes is one of the leading causes of morbidity and mortality, affecting more than 382 million people globally and is projected to double by 2030 [2]. The global burden of disease study reported that over the last two decades NCDs have substantially increased in terms of absolute number of years of life lost and years lived with disability, and diabetes contributing to the largest increase in diseases burden [3]. The most common pathologies caused by diabetes include, microvascular complications such as diabetic retinopathy, diabetic nephropathy leading to chronic kidney diseases, and diabetic neuropathy; and macrovascular complications such as coronary heart disease, and stroke. The aim of this paper is to briefly discuss the increasing burden of diabetes of diabetes in Bangladesh and explore the potential role of information technology to address this issue.

Almost one-fifth of the world's total population with diabetes live in Southeast Asia region, where the prevalence of diabetes is estimated to increase by $71 \%$ by 2035 [2]. The International Diabetes Federation (IDF) Diabetes Atlas indicates that by $2030,10.2 \%$ of the adult population in this region will have diabetes increasing the number of people with diabetes to 120.9 million [4]. Rapid urbanization and economic growth in many countries of Southeast Asia have led to exposure to new risk factors, such as adverse dietary pattern, sedentary lifestyles, obesity, environmental exposure among others in addition to traditional risk factors of increasing age and genetic predisposition [5]. Southeast Asians develop type 2 diabetes at a lower body mass index (BMI) compared to Caucasians and have more central obesity [6]. Moreover, diabetes and its complications also develop at a relative younger age in South Asians compared to the Western population $[7,8]$.

According to the IDF Diabetes Atlas, there are an estimated 8.4 million people with diabetes in Bangladesh and a similar number of people with prediabetes. The IDF projected that the number of people with diabetes will increase to 16.8 million by 2030 , placing Bangladesh among the top ten countries globally in terms of number of people living with diabetes [2]. A scoping review of the increasing trend of diabetes prevalence in Bangladesh showed that the prevalence of diabetes varied from $4.5 \%$ to $35 \%$ with a pooled prevalence of $7.4 \%$ (95\% CI 7.17 7.63) [9]. Another systematic review of the risk factors for diabetes in Bangladesh identified the most common risk factors for diabetes in Bangladesh, namely increased age, obesity, social class, hypertension, family history, sedentary life style among others [6]. Diabetes is a complex condition and impacts on individuals, families, societies, health systems and the nation as a whole. The economic and human costs provoked by diabetes in a large population such as in Bangladesh will be substantial [10]. However, the social and economic impact of diabetes in Bangladesh, as well as its impact on individual, society and health systems level is largely not known. Patients with diabetes lack proper knowledge of their conditions [11], and also suffers from high prevalence of co-morbid depression in Bangladesh $[12,13]$.

The growing epidemic of diabetes in Bangladesh and other developing countries suggest that traditional methods for diabetes management in hospital settings or clinics might not be adequate to control the epidemic alone. Globally, mobile phone technologies have emerged as an essential tool for strengthening health systems and improving disease management in many countries [14]. In recent years, the Government of Bangladesh and the World Health Organization (WHO) have adopted information technologies for health to their strategic plans [15]. Previous studies showed the effectiveness of SMS for increasing medication adherence and behavior changes in developing countries $[16,17]$. Substantial evidence suggest that mobile phones might be a potential tool for addressing NCDs requiring lifelong treatment in developing country settings $[18,19]$. However, such innovative technologies for health interventions are complex and require solid evidence before they could be scaled up. It is high time for the Governments and stakeholders to consider using innovative information technology for prevention and management of diabetes.

\section{References}

1. Islam SM, Purnat TD, Phuong NT, Mwingira U, Schacht K, et al. (2014) Noncommunicable diseases (NCDs) in developing countries: a symposium report. Global Health 10: 81. [Crossref]

Correspondence to: M Shariful Islam, Senior Research Investigator, NonCommunicable Diseases Initiative, ICDDR,B Postdoc Research Fellow, The George Institute for Global Health, Research Tutor, MD Program, Sydney Medical School, University of Sydney, Missenden Road, Camperdown, NSW 2050, Australia, Tel: +61 0405105012; E-mail: Shariful.islam@icddrb.org

Received: November 10, 2016; Accepted: December 20, 2016; Published: December 23, 2016 
2. International Diabetes Federation (2013) IDF Diabetes Atlas (6th edn) Brussels, Belgium: International Diabetes Federation.

3. Murray CJ, Lopez AD (2013) Measuring the global burden of disease. NEJM 369: 448457. [Crossref]

4. International Diabetes Federation (2013) What we do: Epidemiology and Prevention: Diabetes Atlas: Regional Overview: South-East Asia: Regional data. [Crossref]

5. Herman WH, Zimmet $\mathrm{P}$ (2012) Type 2 diabetes: an epidemic requiring global attention and urgent action. Diabetes Care 35: 943-944. [Crossref]

6. Sal-sabil T, Islam A, Shariful Islam SM (2016) Risk Factors for Type 2 Diabetes in Bangladesh: A Systematic Review. Journal of Diabetology 2. [Crossref]

7. Islam SMS, Alam DS, Wahiduzzaman M (2014) Clinical characteristics and complications of patients with type 2 diabetes attending an urban hospital in Bangladesh. Diabetes \& Metabolic Syndrome: Clinical Research \& Reviews. [Crossref]

8. Misra A, Ramchandran A, Jayawardena R, Shrivastava U, Snehalatha C (2014) Diabetes in South Asians. Diabet Med 31: 1153-1162. [Crossref]

9. Biswas T, Islam A, Rawal LB, Islam SM (2016) Increasing prevalence of diabetes in Bangladesh: a scoping review. Public Health 138: 4-11. [Crossref]

10. Shariful Islam SM, Lechner A, Ferrari U, Froeschl G, Niessen LW, et al. (2013) Social and economic impact of diabetics in Bangladesh: protocol for a case-control study. BMC Public Health 13: 1217. [Crossref]

11. Shariful Islam S, Niessen LW, Seissler J, Uta Ferrari, Tuhin Biswas, et al. (2015) Diabetes knowledge and glycemic control among patients with type 2 diabetes in Bangladesh. Springer Plus 4: 284. [Crossref]
12. Islam SMS, Rawal LB, Niessen LW (2015) Prevalence of depression and its associated factors in patients with type 2 diabetes: A cross-sectional study in Dhaka, Bangladesh. Asian Journal of Psychiatry 17: 36-41. [Crossref]

13. Islam SMS, Ferrari U, Seissler J, Niessen L, Lechner A (2015) Association between depression and diabetes amongst adults in Bangladesh: a hospital based case-control study. Journal of Global Health 5: 2. [Crossref]

14. Labrique A, Vasudevan L, Chang LW, Mehl G (2013) H pe for mHealth: More "y" or "o" on the horizon? International Journal of Medical Informatics 82: 467-469.

15. Islam SMS, Tabassum R (2015) Implementation of information and communication technologies for health in Bangladesh. Bulletin of the World Health Organization. [Crossref]

16. Cole-Lewis H, Kershaw T (2010) Text messaging as a tool for behavior change in disease prevention and management. Epidemiologic Reviews 32: 56-69. [Crossref]

17. Free C, Phillips G, Galli L, Louise Watson, Lambert Felix, et al. (2013) The effectiveness of mobile-health technology-based health behaviour change or disease management interventions for health care consumers: a systematic review. PLoS Medicine 10: e1001362. [Crossref]

18. Peiris D, Praveen D, Johnson C, Mogulluru K (2014) Use of mHealth Systems and Tools for Non-Communicable Diseases in Low-and Middle-Income Countries: a Systematic Review. Journal of Cardiovascular Translational Research 7: 677-691. [Crossref]

19. Islam SMS, Niessen LW, Ferrari U, Ali L, Seissler J, et al. (2015) Effects of Mobile Phone SMS to ImproveGlycemic Control Among Patients With Type 2 Diabetes in Bangladesh: A Prospective, Parallel-Group,Randomized Controlled Trial. Diabetes Care 2015: 112-113. [Crossref]

Copyright: (C2016 Islam SMS. This is an open-access article distributed under the terms of the Creative Commons Attribution License, which permits unrestricted use, distribution, and reproduction in any medium, provided the original author and source are credited. 